Supporting Information for:

\title{
SIMULATION OF TRANSIENT PHOTOCONDUCTION IN ORGANIC P-N JUNCTION
}

\section{BI-LAYER PHOTODIODES}

\section{Li Tan, ${ }^{\text {a) }}$ M. David Curtis ${ }^{* a, b)}$, and A. H. Francis ${ }^{\text {b) }}$}

a) Macromolecular Science \& Engineering Center, The University of Michigan, Ann Arbor, Michigan 48109-1055

b) Department of Chemistry, The University of Michigan, Ann Arbor, Michigan 48109-1055

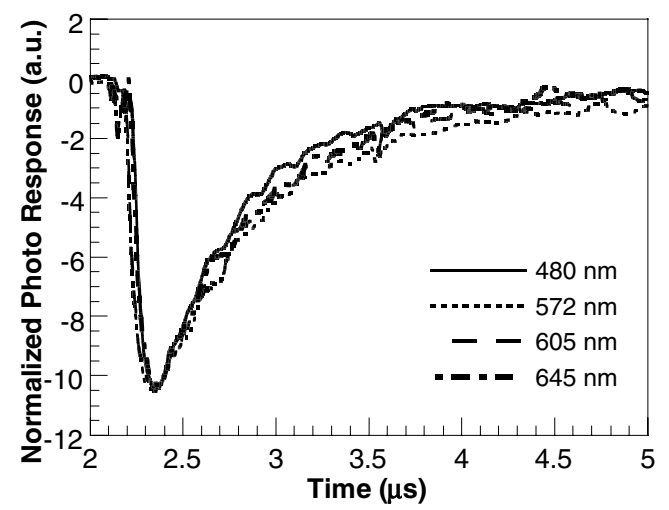

Figure 1S. Normalized transient photoresponses of ITO/P3BT-co-EDOT/PV/Ag: (bottom) $\mathrm{V}_{\mathrm{a}}=-1.0 \mathrm{~V}, \lambda_{\mathrm{exc}}=480,572,605$, and $645 \mathrm{~nm}$. 

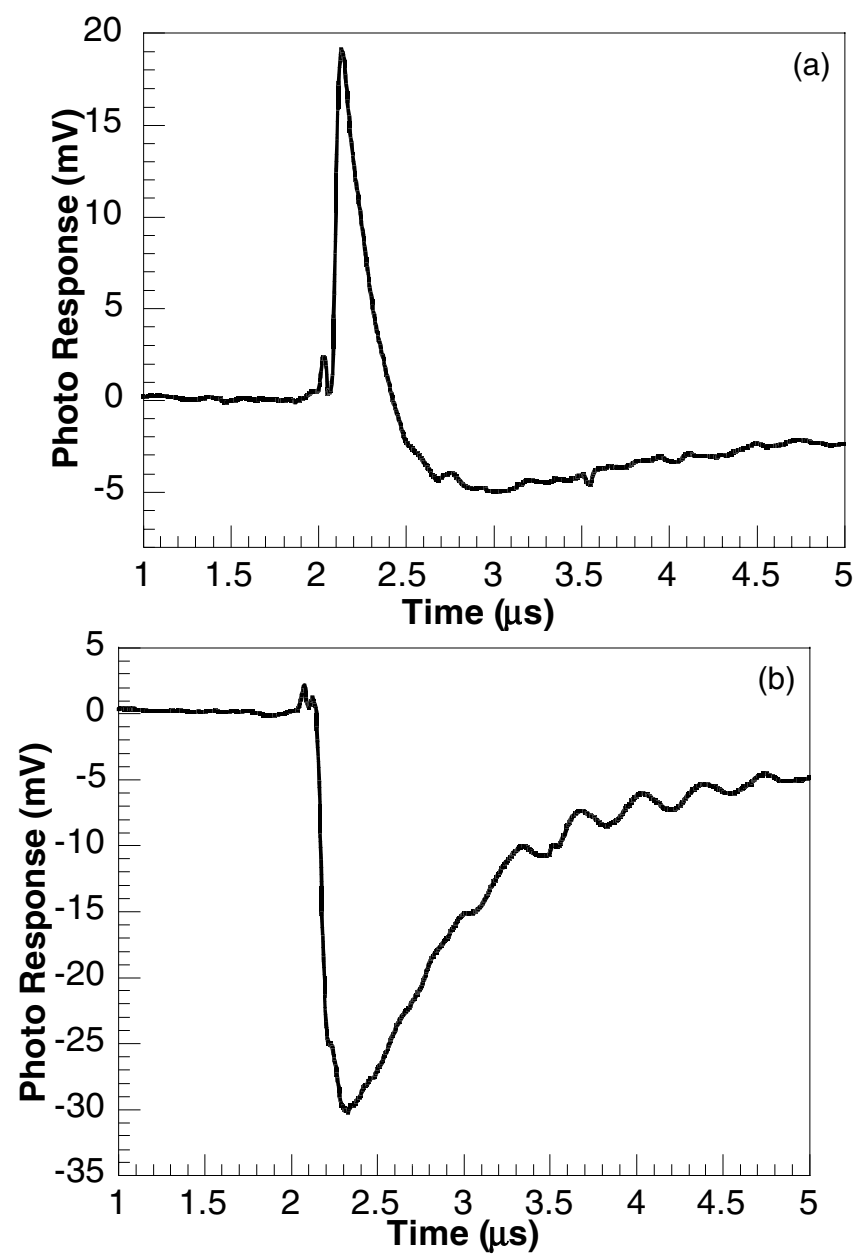

Figure 2S. Photoelectron ejection from Ag electrode in ITO/P3BT-co-EDOT/PV/Ag bi-layer device with $\mathrm{N}_{2}$ laser illumination $\left(\lambda=337 \mathrm{~nm}\right.$ ): (a) $\mathrm{V}_{\mathrm{a}}=0.0 \mathrm{~V}$; (b) positive spike was minimized when reverse bias was applied $\left(V_{a}=-0.18 \mathrm{~V}\right)$.

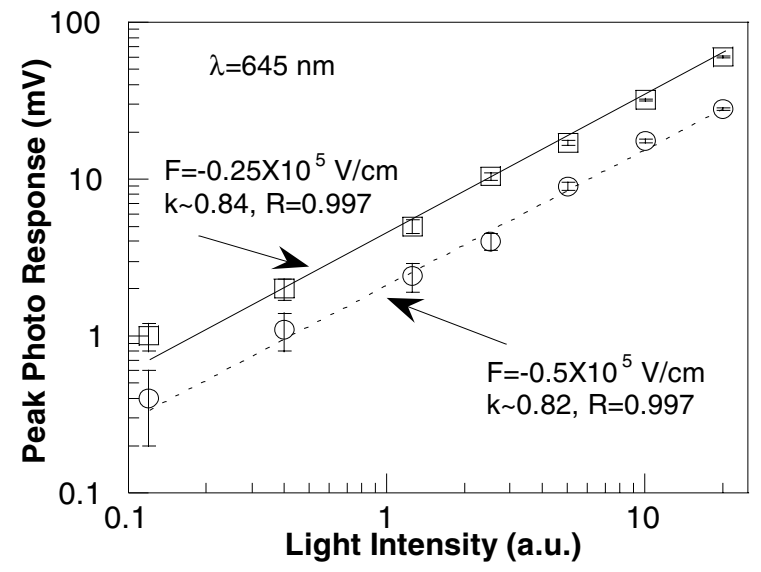

Figure 3S. Dependence of peak transient photo response (ITO/P3BT-co-EDOT/PV/Ag) on the intensity of the illumination $(\lambda=645 \mathrm{~nm})$ under different applied biases: $\mathrm{F}_{\mathrm{a}}=-2.5 \times 10^{4}$, and $-5 \times 10^{4} \mathrm{~V} / \mathrm{cm}$. 


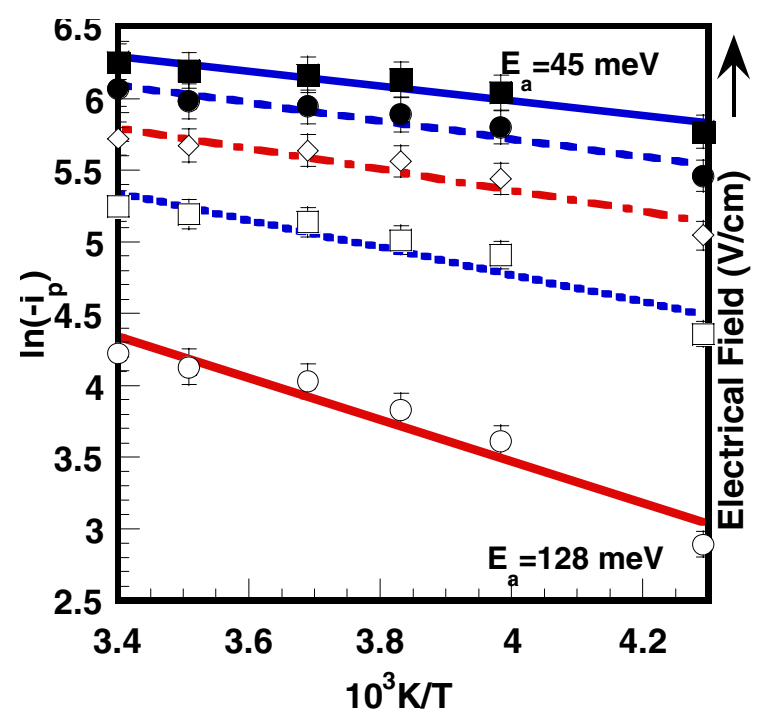

Figure 4S. Plot of $\ln \left(-\mathrm{i}_{\mathrm{p}}\right)$ vs $1 / \mathrm{T}$ at different applied biases: from bottom to top, $\mathrm{V}_{\mathrm{a}}=0.0$, $-0.5,-1.0,-1.5$, and $-2.0 \mathrm{~V}$.

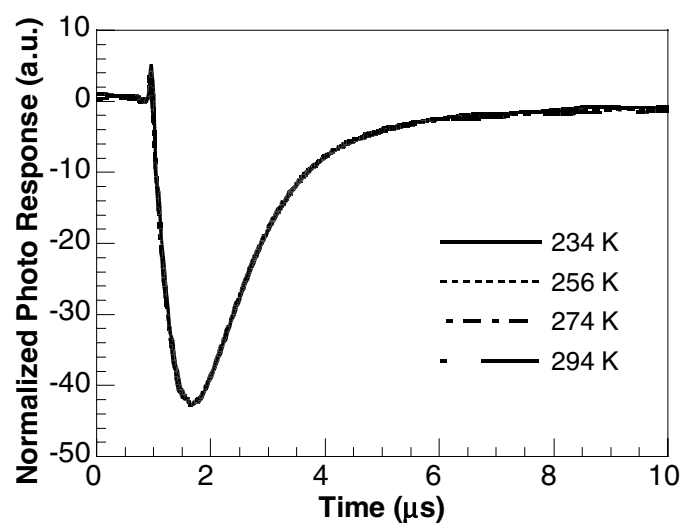

Figure 5S. Normalized transient photo responses of ITO/P3BT-co-EDOT/PV/Ag at different temperatures with illumination from ITO side $(R C=0.6 \mu s)$ (top). 


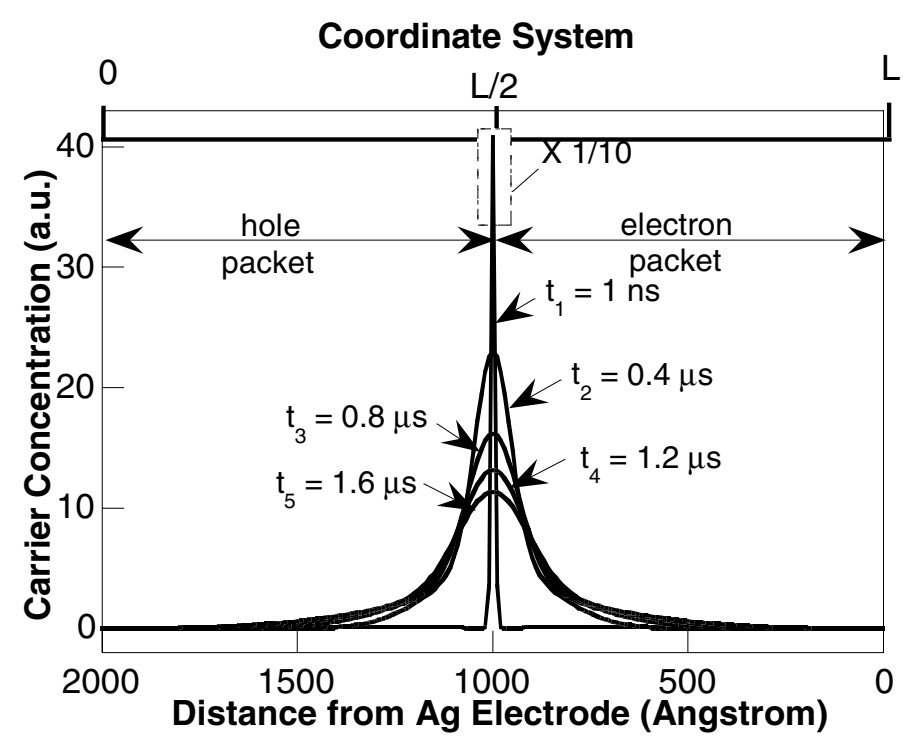

Figure 6S. Simulated carrier diffusion in ITO/polymer/PV/Ag after pulsed illumination., $\mu_{\mathrm{v}}=\mu_{\mathrm{n}}=10^{-4} \mathrm{~cm}^{2} / \mathrm{Vs}$ and $\tau_{\mathrm{n}}=\tau_{\mathrm{v}}=0.1 \mathrm{~ms}$ are assumed.
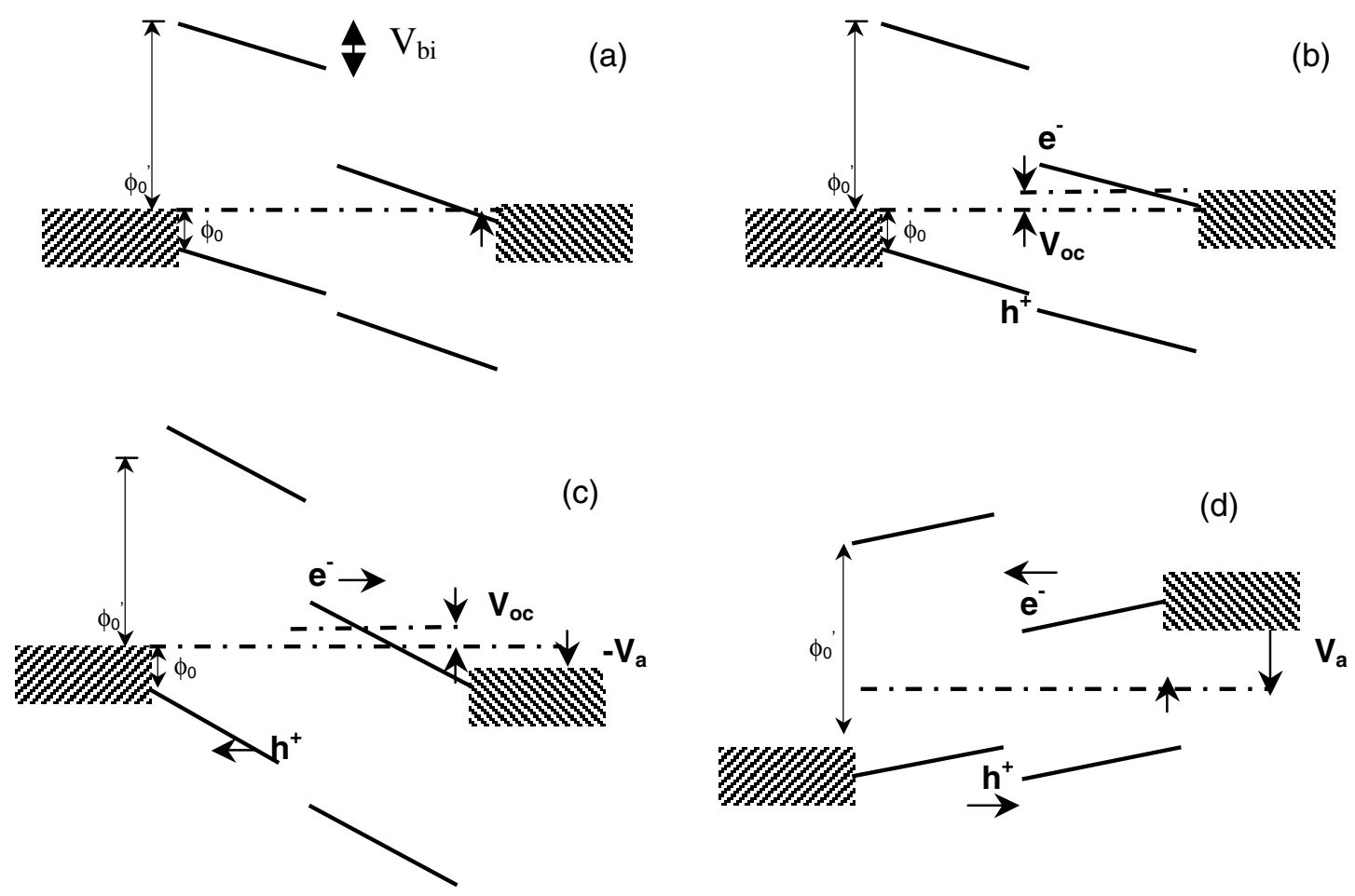

Figure 7S. Energy diagram of the bi-layer photodiode: a) open-circuit (the built-in voltage $\left(V_{b i}\right)$ is produced by the difference in work functions of the electrodes; (b) photocarrier generation at organic/organic interface generates the open circuit voltage, $\left.\mathrm{V}_{\mathrm{oc}} ; \mathrm{c}\right)$ reverse applied bias, $-V_{a}$; and d) forward applied bias, $V_{a}$. 


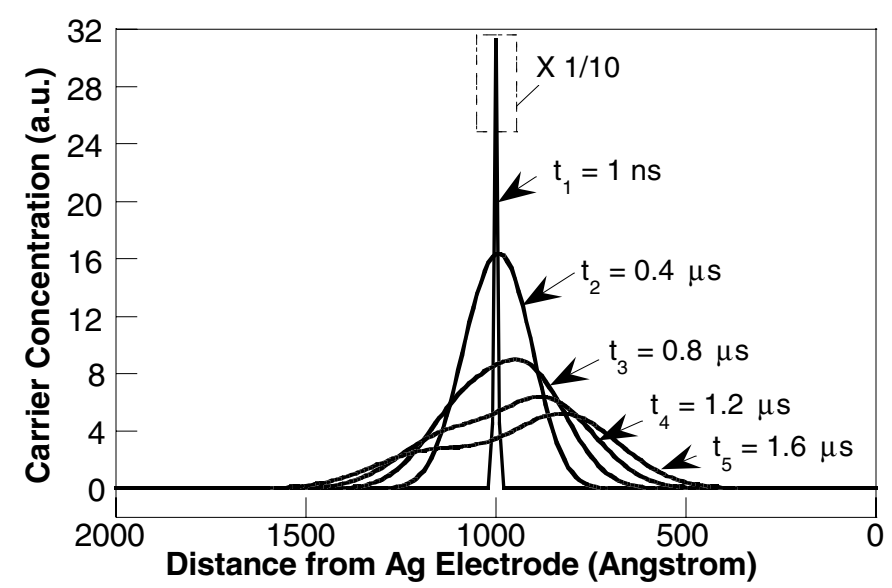

Figure 8S. Simulated carrier diffusion and drift process with different carrier lifetime $\left(\tau_{n}=10^{-5} s\right.$ and $\left.\tau_{p}=10^{-6} s\right) . E_{a}=2 \times 10^{4} V / c m$ and $\mu_{\mathrm{n}}=\mu_{\mathrm{p}}=5 \times 10^{-5} \mathrm{~cm}^{2} / \mathrm{Vs}$ are assumed.

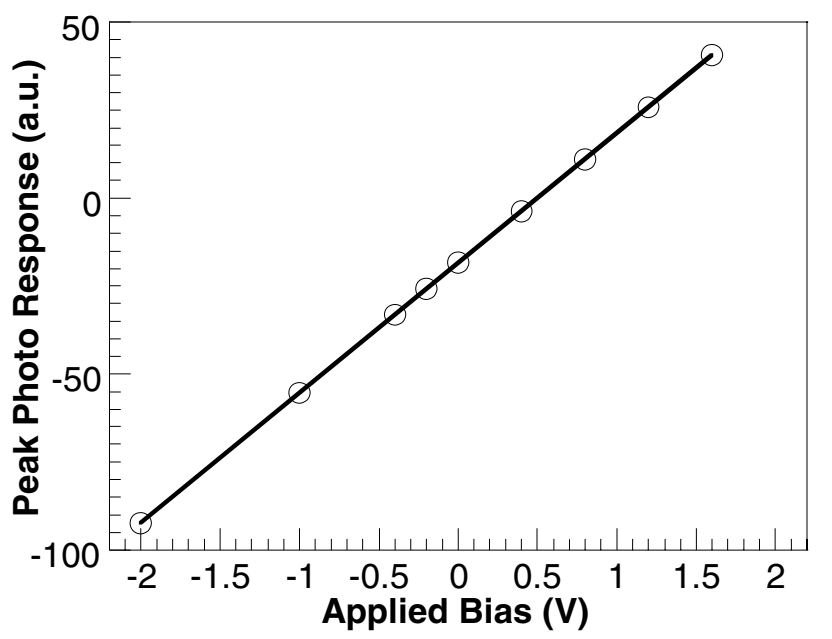

Figure 9S. Plot of the calculated transient current peak heights from Figure 7 vs. applied field. 

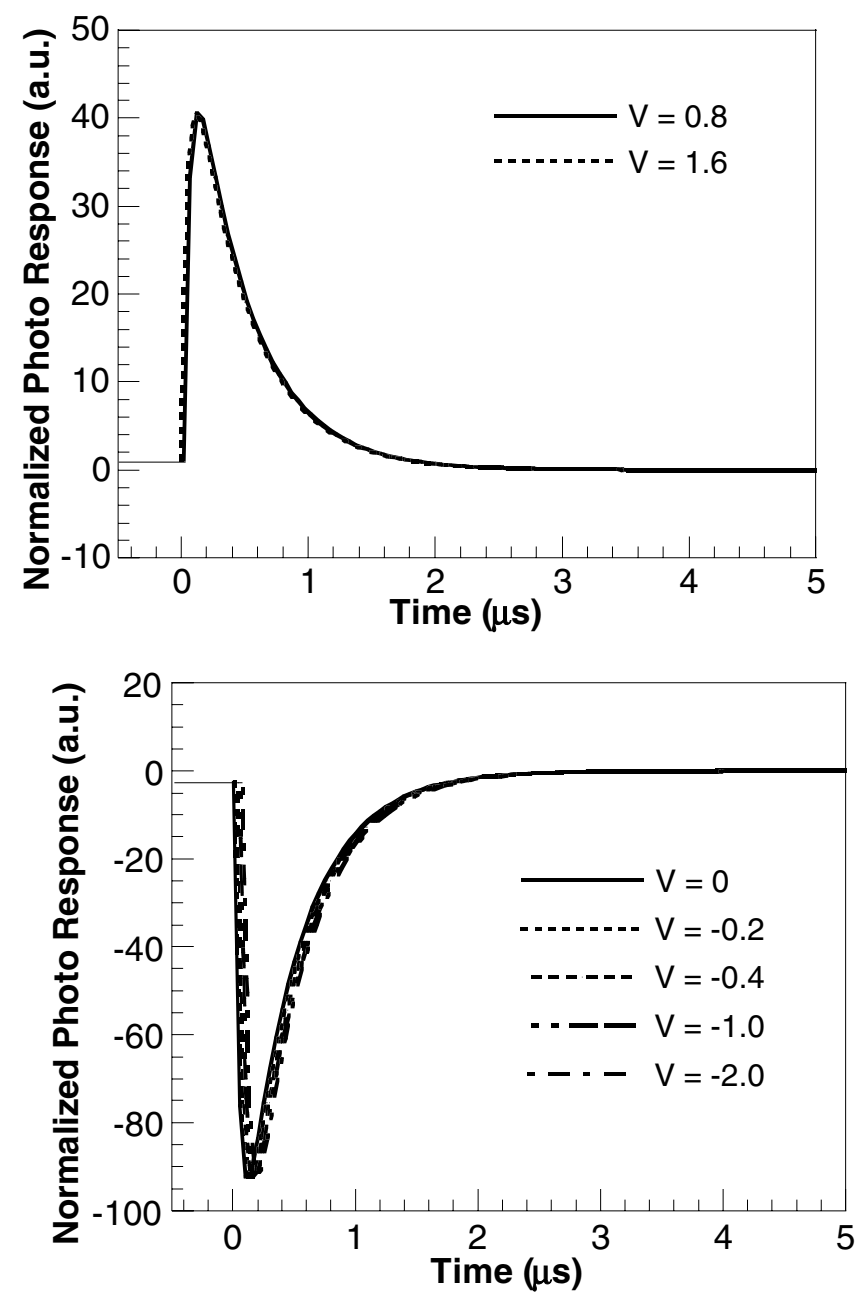

Figure 10S. Simulated transient photo response (normalized) under electric field (reverse or forward bias, $E=10^{4} \sim 10^{5} \mathrm{~V} / \mathrm{cm}$ ) after pulse illumination: $\mu_{p} \sim \mu_{n}=10^{-6} \mathrm{~cm}^{2} / \mathrm{Vs}$ and $\tau_{\mathrm{p}}=\tau_{\mathrm{n}}=4.5 \times 10^{-7} \mathrm{~s}$. (top) forward bias; (bottom) reverse bias and short-circuit. 
LIMITED BOUNDARY SOLUTION-BASIC PROGRAM

FOR SIMULATION OF CURRENT-TIME CURVES OF TRANSIENT PHOTORESPONSE

CLS

DIM MATCARRIER $(201,12)$

DIM MATCURRENT $(201,3)$

$\mathrm{PI}=3.1415926 \#$

$\mathrm{Q}=.000001$

colr $=7$

TMax $=1$ 'time window for carrier distribution

$\mathrm{ZOOM}=200000$

INPUT "CARRIER FILE NAME (MAX 8 CHARACTERS) = ", NAME1\$

INPUT "CURRENT FILE NAME (MAX 8 CHARACTERS $)=$ ", NAME2\$

CLS

$\mathrm{E}=10000$

$\mathrm{Ebi}=2.5 * 10000$

$\mathrm{UH}=.000001$

$\mathrm{UE}=.000001$

$\mathrm{DH}=.0259 * \mathrm{UH} \quad$ 'Einstein Relationship

$\mathrm{DE}=.0259 * \mathrm{UE}$

TAUH $=.00000045 \#$

TAUE $=.00000045 \#$

\section{SCREEN 9}

LOCATE 2, 5: PRINT "E (V/cm) =", E

LOCATE 3, 5: PRINT "HOLE MOBILITY $\left(\mathrm{cm}^{\wedge} 2 / \mathrm{Vs}\right)="$, UH

LOCATE 4, 5: PRINT "ELECTRON MOBILITY $\left(\mathrm{cm}^{\wedge} 2 / \mathrm{Vs}\right)="$, UE

LOCATE 5, 5: PRINT "HOLE DIFFUSION CONSTANT =", DH

LOCATE 6, 5: PRINT "ELECTRON DIFFUSION CONSTANT =", DE

LOCATE 7, 5: PRINT "Tau-h=", TAUH

LOCATE 8, 5: PRINT "Tau-e=", TAUE

$\operatorname{PSET}(0,300)$

LINE -(600, 300), colr

$\operatorname{PSET}(0,300)$

colr $=1$

$\mathrm{L}=.00002$ 
$\mathrm{S}=0^{\prime}$ HOLE CONCENTRATION

$\mathrm{SS}=0^{\prime}$ ELECTRON CONCENTRATION

PSET $(0,300)$

$\mathrm{J}=1$

FOR $X=0$ TO 1 STEP .005

MATCARRIER $(J, 1)=X * 2000$ 'unit:angstrom

$\mathrm{J}=\mathrm{J}+1$

NEXT X

$\mathrm{J}=1$

$\mathrm{K}=1$

STEPCOUNT $=$ TMax $/ 5$

FOR T $=.001$ TO TMax STEP STEPCOUNT

$\mathrm{K}=\mathrm{K}+1$

$\mathrm{SUM}=0$

FOR $X=0$ TO 1 STEP .005

FOR $n=0$ TO 200

$\mathrm{M}=\mathrm{n}$

$\mathrm{S}=(-1) \wedge \mathrm{M} * \mathrm{SIN}((2 * \mathrm{n}+1) * \mathrm{PI} *(\mathrm{X} * \mathrm{~L}-\mathrm{UH} *(\mathrm{E}+\mathrm{Ebi}) * \mathrm{~T} * .000001) / \mathrm{L})$

$* \operatorname{EXP}(-((1 / \mathrm{TAUH})+\mathrm{DH} *((2 * \mathrm{n}+1) * \mathrm{PI} / \mathrm{L}) \wedge 2) * \mathrm{~T} * .000001)$

$\mathrm{SS}=(-1)^{\wedge} \mathrm{M} * \mathrm{SIN}((2 * \mathrm{n}+1) * \mathrm{PI} *(\mathrm{X} * \mathrm{~L}+\mathrm{UE} *(\mathrm{E}+\mathrm{Ebi}) * \mathrm{~T} * .000001) /$

$\mathrm{L}) * \operatorname{EXP}(-((1 / \mathrm{TAUE})+\mathrm{DE} *((2 * \mathrm{n}+1) * \mathrm{PI} / \mathrm{L}) \wedge 2) * \mathrm{~T} * .000001)$

$\mathrm{SUM}=\mathrm{SUM}+(\mathrm{S}+\mathrm{SS}) * 2 / \mathrm{L}$

NEXT $n$

IF SUM $<0$ THEN SUM $=0$

$\mathrm{Y}=300-2 * \mathrm{SUM} * \mathrm{~L}$

$\mathrm{XX}=600 * \mathrm{X}$

LINE -(XX, Y), colr

$\operatorname{MATCARRIER}(\mathrm{J}, \mathrm{K})=\mathrm{SUM} * \mathrm{~L}$

$\mathrm{J}=\mathrm{J}+1$

$S=0$

$\mathrm{SS}=0$

$\mathrm{SUM}=0$

NEXT X

$\mathrm{J}=1$

$\operatorname{PSET}(0,300)$

colr $=$ colr +1

NEXT T

OPEN NAME1 $\$$ FOR OUTPUT AS \#1

FOR $\mathrm{J}=1$ TO 201

PRINT \#1, USING " \#\#\#.\#\#\#\# "; MATCARRIER(J, 1); MATCARRIER(J, 2);

MATCARRIER(J, 3); MATCARRIER(J, 4); MATCARRIER(J, 5); MATCARRIER(J, 6)';

MATCARRIER(J, 8); MATCARRIER(J, 9); MATCARRIER(J, 10) 
NEXT J

CLOSE \#1

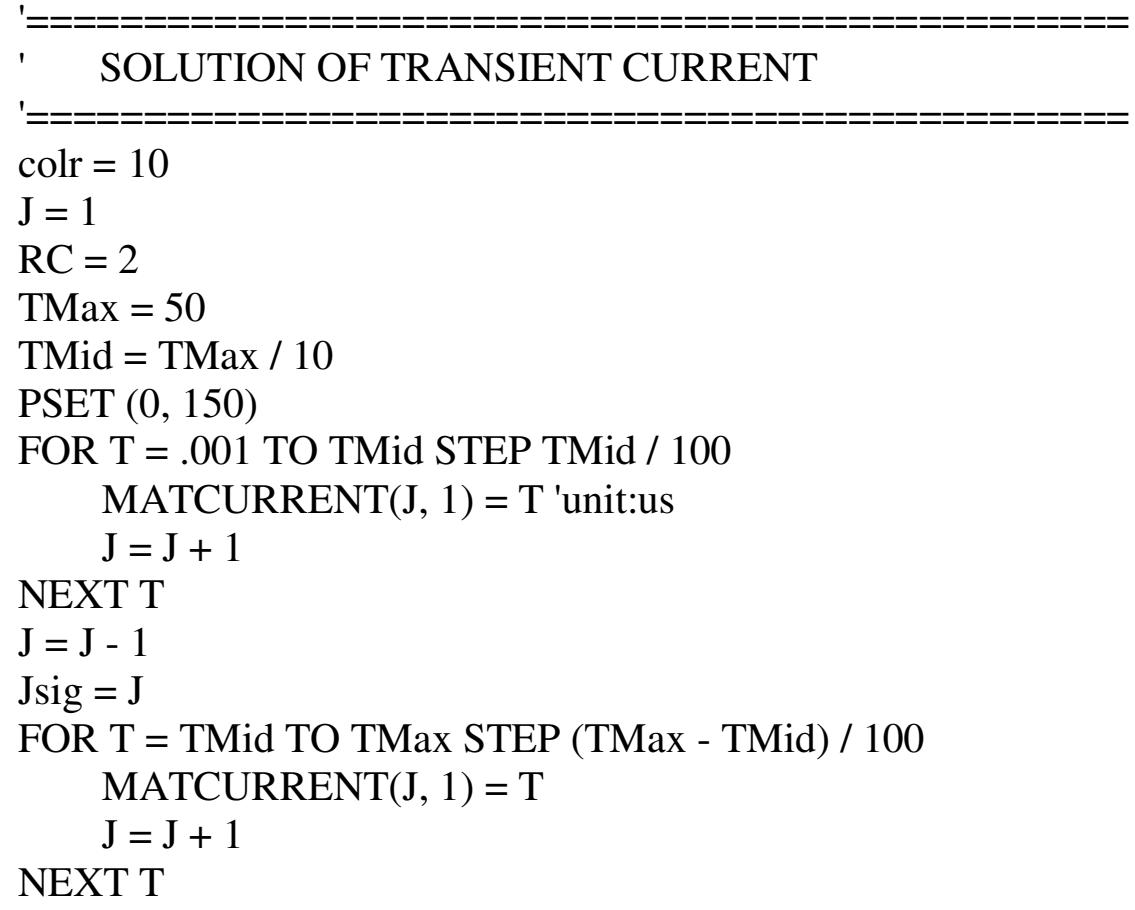

$\mathrm{J}=1$

FOR T $=.001$ TO TMid STEP TMid $/ 100$

DRIFHOLE $=0$

DIFFHOLE $=0$

CURRENTHOLE $=0$

DRIFELEC $=0$

DIFFELEC $=0$

CURRENTELEC $=0$

CURRENT $=0$

FOR $X=0$ TO 1 STEP .01

FOR $n=0$ TO 20

$\mathrm{M}=\mathrm{n}$

$\mathrm{S}=(-1)^{\wedge} \mathrm{M} * \mathrm{SIN}((2 * \mathrm{n}+1) * \mathrm{PI} *(\mathrm{X} * \mathrm{~L}-\mathrm{UH} *(\mathrm{E}+\mathrm{Ebi}) * \mathrm{~T} * .000001) / \mathrm{L})$

$* \operatorname{EXP}\left(-\left((1 / \mathrm{TAUH})+\mathrm{DH} *((2 * \mathrm{n}+1) * \mathrm{PI} / \mathrm{L})^{\wedge} 2\right) * \mathrm{~T} * .000001\right)$

$\mathrm{S} 1=(-1) \wedge \mathrm{M} *((2 * \mathrm{n}+1) * \mathrm{PI}) * \operatorname{COS}((2 * \mathrm{n}+1) * \mathrm{PI} *(\mathrm{X} * \mathrm{~L}-\mathrm{UH} *(\mathrm{E}+$

$\mathrm{Ebi}) * \mathrm{~T} * .000001) / \mathrm{L}) * \operatorname{EXP}(-((1 / \mathrm{TAUH})+\mathrm{DH} *((2 * \mathrm{n}+1) * \mathrm{PI} / \mathrm{L}) \wedge 2) * \mathrm{~T} * .000001)$

$\mathrm{SS}=(-1)^{\wedge} \mathrm{M} * \mathrm{SIN}((2 * \mathrm{n}+1) * \mathrm{PI} *(\mathrm{X} * \mathrm{~L}+\mathrm{UE} *(\mathrm{E}+\mathrm{Ebi}) * \mathrm{~T} * .000001) /$

$\mathrm{L}) * \operatorname{EXP}(-((1 / \mathrm{TAUE})+\mathrm{DE} *((2 * \mathrm{n}+1) * \mathrm{PI} / \mathrm{L}) \wedge 2) * \mathrm{~T} * .000001)$

$\mathrm{SS} 1=(-1)^{\wedge} \mathrm{M} *((2 * \mathrm{n}+1) * \mathrm{PI}) * \mathrm{COS}((2 * \mathrm{n}+1) * \mathrm{PI} *(\mathrm{X} * \mathrm{~L}+\mathrm{UE} *(\mathrm{E}+$

$\mathrm{Ebi}) * \mathrm{~T} * .000001) / \mathrm{L}) * \mathrm{EXP}(-((1 / \mathrm{TAUE})+\mathrm{DE} *((2 * \mathrm{n}+1) * \mathrm{PI} / \mathrm{L}) \wedge$ 2) $* \mathrm{~T} * .000001)$

DRIFHOLE $=$ DRIFHOLE $+\mathrm{S} *(2 / \mathrm{L})$

DIFFHOLE $=$ DIFFHOLE $+\mathrm{S} 1 *(2 / \mathrm{L})$ 


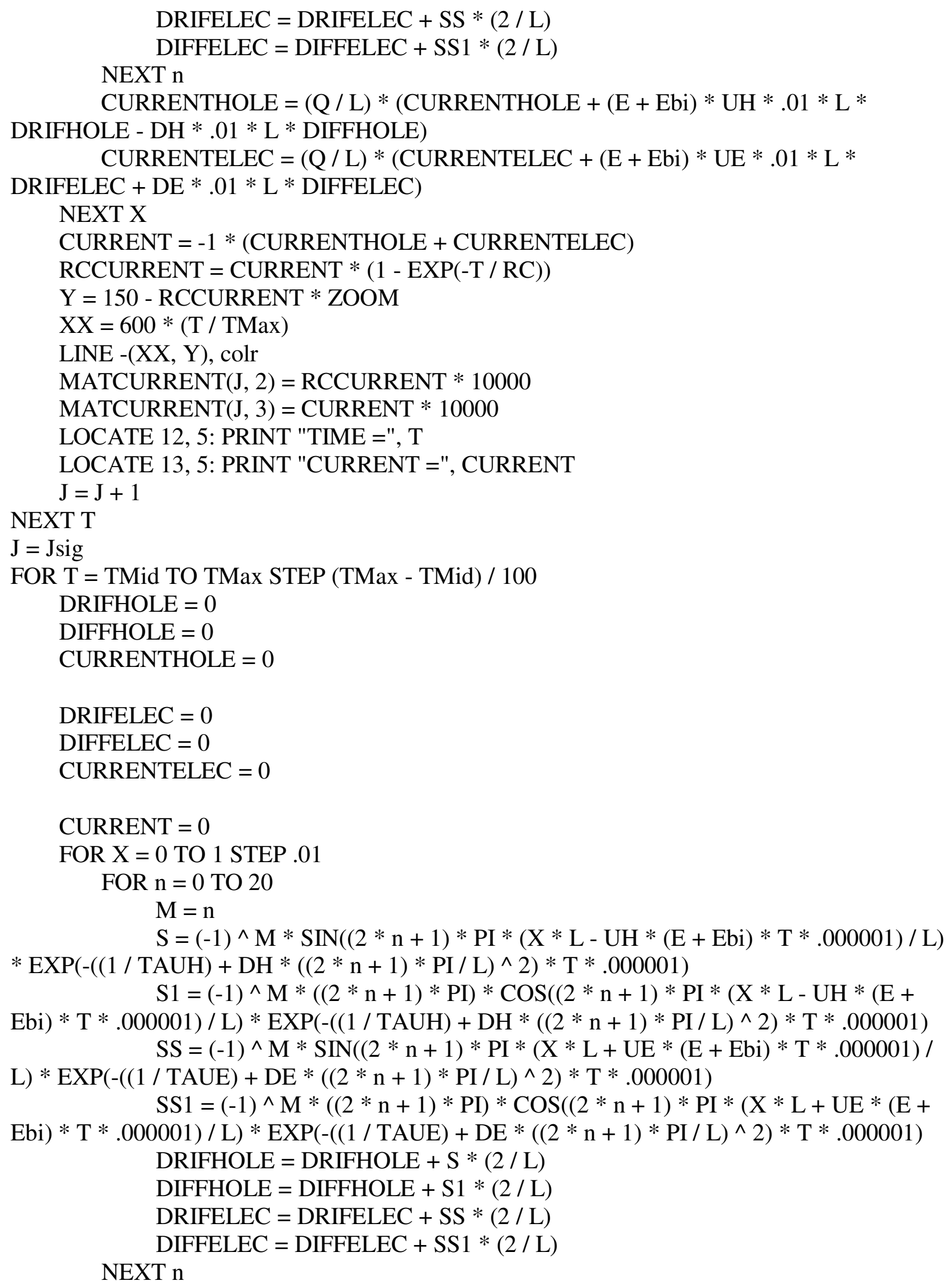




$$
\text { CURRENTHOLE }=(\mathrm{Q} / \mathrm{L}) *(\mathrm{CURRENTHOLE}+(\mathrm{E}+\mathrm{Ebi}) * \mathrm{UH} * .01 * \mathrm{~L} *
$$

DRIFHOLE - DH *.01* L* DIFFHOLE)

CURRENTELEC $=(\mathrm{Q} / \mathrm{L}) *($ CURRENTELEC $+(\mathrm{E}+\mathrm{Ebi}) * \mathrm{UE} * .01 * \mathrm{~L} *$

DRIFELEC $+\mathrm{DE} * .01 * \mathrm{~L} *$ DIFFELEC)

NEXT X

CURRENT $=-1 *($ CURRENTHOLE + CURRENTELEC $)$ 'the negative sign represents the current direction in the external circuit

RCCURRENT $=$ CURRENT $*(1-$ EXP $(-\mathrm{T} / \mathrm{RC}))$

$\mathrm{Y}=150-$ RCCURRENT $*$ ZOOM

$\mathrm{XX}=600 *(\mathrm{~T} / \mathrm{TMax})$

LINE - $(\mathrm{XX}, \mathrm{Y})$, colr

MATCURRENT $(\mathrm{J}, 2)=$ RCCURRENT $* 10000$

MATCURRENT $(\mathrm{J}, 3)=$ CURRENT $* 10000$

LOCATE 12, 5: PRINT "TIME $=", \mathrm{~T}$

LOCATE 13, 5: PRINT "CURRENT =", CURRENT

$\mathrm{J}=\mathrm{J}+1$

NEXT T

$\operatorname{PSET}(0,300)$

OPEN NAME2\$ FOR OUTPUT AS \#2

FOR J $=1$ TO 200

PRINT \#2, USING " \#\#\#.\#\#\#\# "; MATCURRENT(J, 1); MATCURRENT(J, 2);

MATCURRENT $(\mathrm{J}, 3)$

NEXT J

CLOSE \#2

END 